\title{
Raw data from Ocular Response Analyzer applied for differentiation of normal and glaucoma patients
}

\author{
AgniesZKa JÓŹWIK ${ }^{1 *}$, Magdalena ASEJCZYK-WIDLICKA ${ }^{1}$, AgNIESZKa BOSZCZYK ${ }^{1}$, \\ HENRYK KASPRZAK ${ }^{1}$, PATRYCJA KRZYŻANOWSKA-BERKOWSKA ${ }^{2}$ \\ ${ }^{1}$ Department of Optics and Photonics, Wrocław University of Science and Technology, \\ Wybrzeże Wyspiańskiego 27, 50-370 Wrocław, Poland \\ ${ }^{2}$ Department of Ophthalmology, Wroclaw Medical University, \\ Wybrzeże Ludwika Pasteura 1, 50-367 Wrocław, Poland \\ ${ }^{*}$ Corresponding author: agnieszka.jozwik@pwr.edu.pl
}

\begin{abstract}
Purpose: Presented study describes new parameters calculated from the Ocular Response Analyzer (ORA) raw data. Such an approach can increase the applicability of the ORA in ophthalmic diagnosis. Among many proposed and examined by us parameters from raw data of the air pressure and applanation curves, only a few were chosen and then applied for characterizing a selected group of patients. Methods: The study included healthy subjects in a control group and patients divided into 2 groups: suspect and glaucoma. A series of four ORA measurements were taken from each subject. The raw ORA data were numerically analyzed and new parameters were calculated from the ORA curves for each measurement. Comparative analysis was carried out for the newly proposed parameters (and original parameters from the ORA device). Results: This interesting finding is that the new parameters showed a statistically significant ability to distinguish the glaucoma suspect group from healthy and glaucomatous patients. Moreover comparable or higher repeatability than for IOPg and CH was obtained. Conclusion: Raw data from the ORA enables definition and numerical analysis of new parameters, characterizing every measurement, which can be successfully used for describing an individual eye and differentiating between some specific groups of patients.
\end{abstract}

Keywords: glaucoma, intraocular pressure, Ocular Response Analyzer, raw data, tonometry.

\section{Introduction}

The value of intraocular pressure (IOP) is a crucial and clinical parameter for the screening, diagnosis and management of glaucoma. Despite many different methods and devices of IOP measurements, the problem of the reliable measurement of IOP is still open and challenging. 
Different results obtained for the same eye may depend on quasi-periodical variations of IOP with blood pulsation [1], as well as on other variations of the biomechanical properties of the anterior eye. Measurements of IOP dynamics taken by the Pascal dynamic contour tonometer (DCT) or the SmartLens dynamic observing tonometer demonstrate IOP pulsations with amplitude in the range of $2-6 \mathrm{mmHg}[\underline{2}, \underline{3}]$. Apart from these dynamical changes, there are some static parameters such as corneal geometry (thickness and curvature) or the individual elastic features of the anterior eye segment that have an influence on the measured value of IOP. The necessary corrections of the obtained IOP results, due to these parameters, should be considered. However, this is not very often taken into account. Goldman tonometry gives the value of IOP without considering corneal thickness. Researchers have shown that central corneal thickness (CCT) affects the pressure value of IOP [늠 $\underline{6}$. Higher values of IOP are observed for higher values of the central corneal thickness. In turn, thinner corneas result in lower IOP readings $[\underline{4}, \underline{5}]$. Correction of corneal thickness during the measurement of IOP is used in commercially available tonometers. Other corneal properties, such as radius of curvature or elastic moduli, could have a more significant influence on the correctness of this measurement $[\underline{7}, \underline{8}]$. Viscoelastic properties of the cornea and the anterior eye, and also its behaviour during measurement, are important and investigated problems. High complexity and dynamical variability of the eye structures and their biomechanical properties make the exact measurement of temporary IOP value difficult.

New generation of tonometers, such as Ocular Response Analyzer (ORA, Reichert Technologies, Depew, NY) and Corneal Visualization Scheimpflug Technology (Corvis ST, Oculus, Wetzlar, Germany) start to evaluate some biomechanical properties of the anterior eye and their correlations with eye pathologies. The ORA is a noninvasive air-puff tonometer that measures the biomechanical response of the eye to the impulse of air [9] and some biomechanical properties of the cornea [10]. The device registers values of the air pressure outgoing from the air jet, and the light intensity, reflected from the corneal apex during corneal deformations. These two parameters are presented in form of two curves given by the device. The second, applanation curve should have two local maxima, representing temporal positions of both corneal applanations, where the intensity of light reflected from the corneal apex is the highest. However, in some cases, the applanation curve shows fast local variations. Therefore, ORA's software smooth this curve to better visualize temporal positions of both applanation times $t_{1 \mathrm{a}}$ and $t_{2 \mathrm{a}}$. Air pressures corresponding to both applanations (inward and outward) are called $P_{1}$ and $P_{2}$ (see Fig. 1).

Next, software of the device analyzes the interactions between these two curves and estimates IOP as well as other parameters describing the anterior eye properties. Apart from the results related to IOP, such as Goldmann-correlated intraocular pressure (IOPg) and corneal-compensated intraocular pressure (IOPcc), the ORA generates two values related to corneal biomechanics: corneal hysteresis $(\mathrm{CH})$ and corneal resistance factor (CRF). Calculation of pressure IOPcc is based on the mutual relationship between the first and the second applanations with appropriate constants and is not di- 
rectly related to the thickness of the cornea [9]. $\mathrm{CH}$ and $\mathrm{CRF}$ can demonstrate specific properties of the anterior segment in case of keratoconus or glaucoma [11-13]. It has been shown that IOPg and IOPcc are higher in the case of glaucoma when $\mathrm{CH}$ and CRF are lower $[\underline{14}, \underline{15}]$. Earlier research showed that $\mathrm{CH}$ and $\mathrm{CRF}$ do not change during the daily cycle. However, intraocular pressure changes throughout the day and is higher in the nocturnal period in comparison to the diurnal one [16]. ORA gives 37 additional parameters that mainly describe the applanation curve, allowing a more detailed analysis of the corneal deformation process. They are not commonly used in ophthalmic practise, but could be more useful in the detection of corneal changes in specific corneal conditions [17-19]. Some authors have implemented new additional parameters to characterize the properties of the cornea $[\underline{11}, \underline{17}, \underline{20}]$. Variability of ORA measurements is associated, to a lesser degree, with the quality of waveform scans [21]. The quality of each measurement is described by a waveform score parameter, ranging between 0 and 10 .

ORA device provides access to raw data of both air pressure and applanation curves allowing application of different numerical procedures in order to define new parameters characterizing the anterior eye. Single measurement on the patient eye gives the already mentioned four basic ORA parameters. However, different measurements on the same eye give less or more different results. The averaged values obtained from these few measurements can be used as characteristic value of obtained parameters. However, as it was shown in the paper [22] there exists some individual dependency between $P_{1}, P_{2}$ pressures and some other raw parameters from different measurements, characteristic for the patient. So, such approach needs a few measurements on a single patient eye to define new characteristic parameters. These parameters, characterizing different measurements (multimeasurements parameters) for the same patient eye, can be also used for differentiations of the eye properties [22]. Despite the differences in the form of these two curves given by the ORA for individual measurements on the same eye, there still exist some characteristic dependences and correlations between selected parameters for the individual eye.

The aim of this study was to propose the new approach for the analysis of ORA raw data and to ascertain how the parameters measured by the Ocular Response Analyzer and those calculated from the raw data can be best utilised to improve the diagnosis of glaucoma and early detection of glaucoma suspects.

\section{Materials and methods}

The prospective study included 338 participants. Only one eye of the patient was measured. Control group included 43 healthy volunteers without any eye disease. Patients were divided into two groups: 107 patients were classified as glaucoma group and 86 subjects as glaucoma suspect group. Patients from the glaucoma suspect group were diagnosed with glaucomatous optic disc appearance after clinical assessment. Patients with normal visual field, open angle and intraocular pressure below or equal $21 \mathrm{mmHg}$, 
but with neuretinal rim narrowing with concentric enlargement, concentric enlargement, localized notch or both, were qualified for this group. Glaucoma group included patients with the primary open angle glaucoma and with the primary angle closure glaucoma. Diagnosis of them was based on glaucomatous changes in the optic nerve head with associated visual field defects. The criteria for exclusion from the study were: any systemic disease, intraocular surgery less than twelve months before the study start date, refractive surgery, conjunctival or intraocular inflammation, corneal abnormalities such as edema or scars, and contact lens wear. In the glaucoma group, patients were taking beta-blocker drops (27\%), prostaglandins (36\%), carbonic anhydrase inhibitor eye drops $(28 \%)$ and alpha agonists (9\%). Twenty-eight percent of patients in the suspect group were taking medications. Patients were taking beta-blocker drops (12\%), prostaglandins $(7 \%)$, carbonic anhydrase inhibitor eye drops (8\%) and alpha agonists (1\%).

All patients were recruited from the Glaucoma Clinic at the Department of Ophthalmology of the Wroclaw Medical University and were informed about the process of measurement, to which they voluntarily agreed. The study had the approval of a local ethical committee (Ethics Committee of the Wroclaw Medical University agreement, KB 503/2011), and the informed consent, according to the tenets of the Declaration of Helsinki, was obtained from each participant before examination.

Each measurement by the Ocular Response Analyzer gives a total of 800 measured points describing air pressure and applanation curves (each 400 data points). The analysis was carried out on results taken from the raw data, exported directly from the device. Both curves were processed using numerical procedures (Matlab, Math Works, Inc., Natick, MA, USA, version 2011a). First, the Gaussian filter (size of window 17 data points) was applied for smoothing both curves. As in our previous work, measurements below 2 in the waveform score quality parameter were rejected from further processing due to their noise [22]. Four consecutive measurements with the waveform score greater than 2 were analyzed.

The raw data and smoothed curves are presented in Fig. 1. Times of the first $t_{1 \mathrm{a}}$ and the second $t_{2 \mathrm{a}}$ applanations, the time of the maximum air pressure curve $t_{\mathrm{m}}$ and the corresponding applanation pressures $P_{1}$ and $P_{2}$, as well as the maximal air pressure MP were numerically calculated. Additionally, value $P_{\mathrm{a}}$ was calculated as the arithmetic average of $P_{1}$ and $P_{2}$.

During the extended numerical analysis of the point positions characterizing both curves and their mutual dependencies, some new parameters were defined (HM, $P_{1} / \mathrm{CCT}$, $\left.P_{\mathrm{a}} / \mathrm{CCT}, P_{\mathrm{a}} / \mathrm{smm}, \mathrm{dm}, b_{1.45}\right)$. The parameter smm was already described previously [를,

$$
\mathrm{smm}=\frac{t_{\mathrm{m}}}{\sqrt{\mathrm{dm}_{1} \mathrm{dm}_{2}}}-1
$$

where $t_{\mathrm{m}}$ - the time of appearance of maximal air pressure curve, $\mathrm{dm}_{1}$ - the time between the first applanation and the maximal air pressure curve, and $\mathrm{dm}_{2}-$ the time between the 


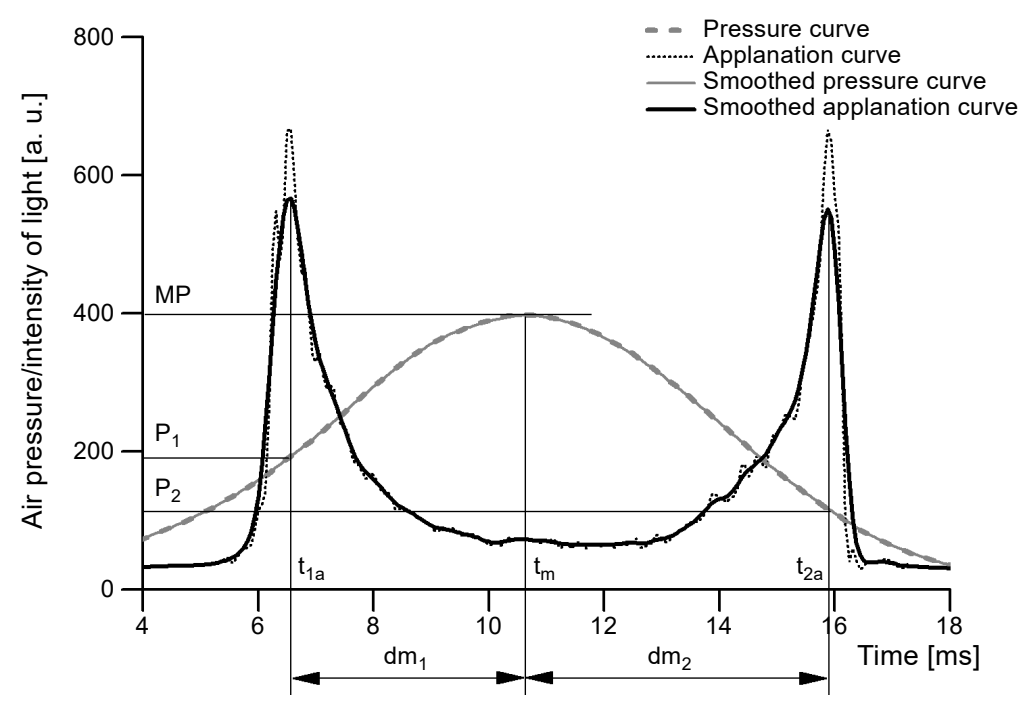

Fig. 1. Data obtained from the Ocular Response Analyzer - raw and smoothed data.

maximal air pressure curve and the second applanation (Fig. 1). Parameter dm specifies the time between two applanations $\left(\mathrm{dm}_{1}+\mathrm{dm}_{2}\right)$. The constant reference value $\mathrm{smm}_{\mathrm{r}}$ was calculated as the arithmetic mean of the smm parameter for all the measurements and the value of $\mathrm{smm}_{\mathrm{r}}=1.45$ was accepted for further analysis. As it was shown in [르] the parameter smm for each patient is strongly correlated with applanation pressures $P_{1}$, $P_{2}$ and their average value $P_{\mathrm{a}}$. Results with a higher correlation were observed and recorded for the dependence between $P_{\mathrm{a}}^{-1 / \mathrm{smm}}$ and smm. Pearson correlation coefficients are included in the range 0.995 to 0.999 (mean value 0.997 , median 0.999 ) for all patients.

For each patient, the multimeasurement parameter $b_{1.45}$ was based on four measurements and calculated from the following equation:

$$
b_{1.45}=A P_{\mathrm{a}}^{-1 / \mathrm{smm}_{\mathrm{r}}}+B
$$

where $A$ and $B$ represent the coefficients of the linear regression of the dependence between $P_{\mathrm{a}}^{-1 / \mathrm{smm}}$ and smm parameters.

Additionally, two parameters: $P_{\mathrm{a}} / \mathrm{smm}$ and $\mathrm{HM}$ (hysteresis modified) are proposed, where $\mathrm{HM}$ is described by the following equation:

$$
\mathrm{HM}=\frac{\mathrm{MP}-P_{2}}{\mathrm{MP}-P_{1}}
$$

where the parameter MP is the maximal value of the air pressure curve. $\mathrm{CH}$ takes into account the difference between pressures $P_{1}$ and $P_{2}$ (Fig. 1), while neglecting the max- 
imal value of the air pressure MP. However, it can occur that the values $P_{1}, P_{2}$ and $\mathrm{CH}$ are respectively the same for the two measurements with the pressure curves having different values of MP. The HM parameter gives different values in both cases. The central corneal thickness CCT was then measured using ultrasonographic pachymetry.

Repeatability and reproducibility of the considered parameters for four measurements on each patient were evaluated by the intraclass correlation coefficient (ICC) and the coefficient of variation (CV). ICCs above 0.75 are usually considered to indicate good repeatability. The statistical analysis of the results for normal, suspect and glaucoma groups was applied. The Shapiro-Wilk test was used to define the distribution of results. Normality was rejected in the majority of cases $(p<0.05)$. Multicomparison for independent groups has been performed using the Kruskal-Wallis test for IOPg, IOPcc, CH, CRF, CCT (Statistica, ver. 14, StatSoft, Inc., USA). To test the new parameters between each pair of groups, the Mann-Whitney test for two independent groups was used. Statistical significance was taken as $p<0.05$.

\section{Results}

The main group demographics together with the group number of subjects, mean age, CCT, IOPg, IOPcc, CH, CRF are shown in Table 1. Statistically significant differences (Kruskal-Wallis test, multicomparison for independent groups, $p<0.05$ ) between the median age, IOPg, $\mathrm{CH}$ and $\mathrm{CRF}$ for all considered groups were obtained.

Proposed new parameters: $\mathrm{HM}, P_{\mathrm{a}} / \mathrm{smm}$, dm, as well as the values of intraocular pressure IOPg and corneal hysteresis $\mathrm{CH}$ were included for analysis. Table 2 shows

$\mathrm{T}$ a b 1 e 1. Number of subjects, mean $( \pm \mathrm{SD})$ and range of age, CCT, IOPg, IOPcc, CH and CRF and the result of the Kruskal $p$-value (ANOVA) statistical difference for each parameter between the groups for the three considered groups. Statistically significant results $(p<0.05)$ are shown in the bold font.

\begin{tabular}{llllr}
\hline & Normal & Suspect & Glaucoma & $p$-value \\
\hline Number (M/F) & $43(13 / 30)$ & $86(25 / 62)$ & $209(58 / 151)$ & \multicolumn{1}{c}{} \\
Age [years] & $73 \pm 11(38-95)$ & $54 \pm 17(20-84)$ & $64 \pm 12(18-82)$ & $<\mathbf{0 . 0 0 1}$ \\
CCT $[\mu \mathrm{m}]$ & $560 \pm 50(447-725)$ & $560 \pm 34(450-665)$ & $552 \pm 38(424-648)$ & $>0.050$ \\
IOPg $[\mathrm{mmHg}]$ & $15.8 \pm 3.4(9.7-23.7)$ & $18.2 \pm 5.5(8.3-45.0)$ & $16.5 \pm 4.5(8.0-41.4)$ & $\mathbf{0 . 0 0 7}$ \\
IOPcc [mmHg] & $16.5 \pm 3.3(10.3-23.9)$ & $17.6 \pm 5.3(10.1-46.8)$ & $16.9 \pm 5.0(6.8-41.3)$ & $>0.050$ \\
CH $[\mathrm{mmHg}]$ & $10.2 \pm 1.4(7.1-13.4)$ & $11.1 \pm 1.7(5.45-14.2)$ & $10.3 \pm 1.9(4.2-16.7)$ & $<\mathbf{0 . 0 0 1}$ \\
CRF $[\mathrm{mmHg}]$ & $10.3 \pm 1.6(7.7-14.9)$ & $11.8 \pm 2.0(7.0-15.7)$ & $10.6 \pm 1.7(5.3-15.8)$ & $<\mathbf{0 . 0 0 1}$ \\
\hline
\end{tabular}

$\mathrm{T} \mathrm{a} \mathrm{b} 1$ e 2. The intraclass correlation coefficients (ICC) and coefficients of variation (CV) [\%] calculated for 5 parameters and also their quotients by corneal central thickness (CCT).

\begin{tabular}{|c|c|c|c|c|c|c|c|c|c|c|}
\hline & IOPg & $\frac{\mathrm{IOPg}}{\mathrm{CCT}}$ & $\mathrm{CH}$ & $\frac{\mathrm{CH}}{\mathrm{CCT}}$ & HM & $\frac{\mathrm{HM}}{\mathrm{CCT}}$ & $\frac{P_{\mathrm{a}}}{\mathrm{smm}}$ & $\frac{P_{\mathrm{a}}}{\mathrm{smm} \cdot \mathrm{CCT}}$ & $\mathrm{dm}$ & $\frac{\mathrm{dm}}{\mathrm{CCT}}$ \\
\hline$\overline{\mathrm{ICC}}$ & 0.91 & 0.92 & 0.69 & 0.69 & 0.57 & 0.88 & 0.83 & 0.99 & 0.86 & 0.96 \\
\hline $\mathrm{CV}$ & 28 & 29 & 17 & 16 & 4 & 9 & 3 & 10 & 6 & 11 \\
\hline
\end{tabular}


the variability of the above 5 parameters and their quotients by corneal central thickness for all patients.

IOPg is characterized by greater repeatability $(\mathrm{ICC}=0.91)$ than $\mathrm{CH}(\mathrm{ICC}=0.69)$. It also has a wider distribution of results in comparison to $\mathrm{CH}$. Both parameters, when divided by corneal thickness, show very similar values to ICC and CV.

The analysis of $P_{\mathrm{a}} /(\mathrm{smm} \cdot \mathrm{CCT})$ shows that this parameter differentiates patients better than IOPg or $\mathrm{CH}$, and its variation $\mathrm{CV}$ is smaller (10\%) than variations of intraocular pressure $\mathrm{IOPg}(28 \%)$ and $\mathrm{CH}(17 \%)$. Similar high values of ICC and low CV are obtained for the parameter $\mathrm{dm} / \mathrm{CCT}$. As was expected, the parameter $\mathrm{HM}$ is strongly correlated with $\mathrm{CH}(r=0.91, p<0.05)$. HM represents similar biomechanical characteristics as $\mathrm{CH}$. However, if corneal thickness is taken into account (HM/CCT), it has higher repeatability than $\mathrm{CH}$ and $\mathrm{CH} / \mathrm{CCT}$.

For further analysis, every parameter measured four times for one patient was averaged. Most of the analyzed parameters, including the intraocular pressure IOPg and the corneal hysteresis $\mathrm{CH}$, differentiate normal-suspect and suspect-glaucoma groups, without registering any differences between the normal and glaucoma groups. This is most likely due to the pharmacological treatment applied for glaucoma patients. Moreover, the parameters $P_{1} / \mathrm{CCT}$ and $P_{\mathrm{a}} / \mathrm{CCT}$ were also considered in the analysis due to a very high ICC being obtained for both parameters ( 0.93 for $P_{1} / \mathrm{CCT}$ and $\left.P_{\mathrm{a}} / \mathrm{CCT}\right)$. The results for four exemplary parameters are presented as box plots (Fig. 2).
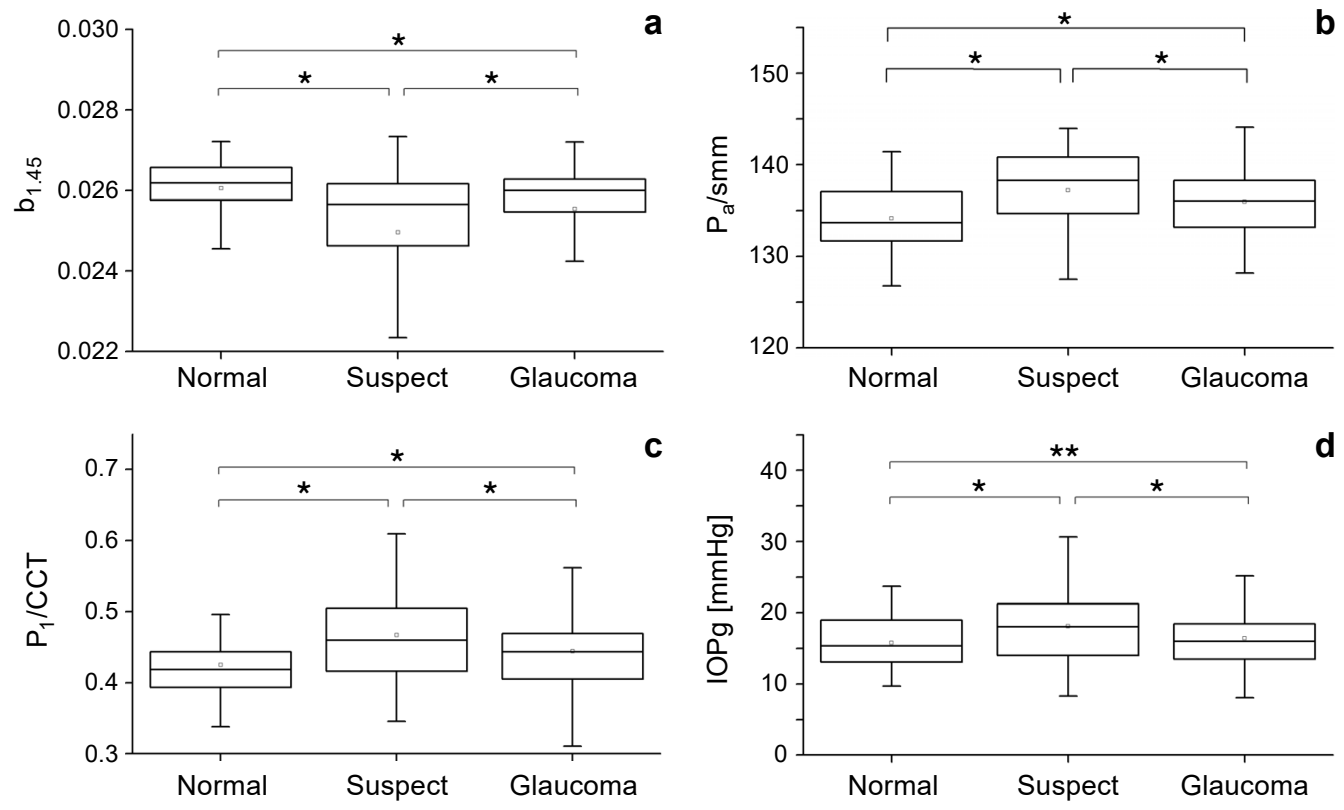

Fig. 2. Box plots of four parameters $b_{1.45}(\mathbf{a}), P_{\mathrm{a}} / \mathrm{smm}(\mathbf{b}), P_{1} / \mathrm{CCT}(\mathbf{c})$, and intraocular pressure $\operatorname{IOPg}(\mathbf{d})$ for patients divided into three groups. Stars represent the interpretation of $p$-values: one star $(*)$ means a statistical significant difference and two stars $(\star \star)$ - no difference between the two compared groups. 
$\mathrm{T} \mathrm{a} \mathrm{b} 1 \mathrm{e} 3$. Mean values $\pm \mathrm{SD}$ for $\mathrm{IOPg}, \mathrm{CH}$ and the new parameters calculated from raw data.

\begin{tabular}{llll}
\hline & Normal & Suspect & Glaucoma \\
\hline$P_{\mathrm{a}} / \mathrm{smm}$ & $134.1 \pm 3.4$ & $137.2 \pm 4.5$ & $135.3 \pm 3.5$ \\
$b_{1.45}$ & $-0.0261 \pm 0.0007$ & $-0.0250 \pm 0.0024$ & $-0.0256 \pm 0.0019$ \\
$P_{\mathrm{a}} / \mathrm{CCT}$ & $0.36 \pm 0.05$ & $0.39 \pm 0.08$ & $0.37 \pm 0.06$ \\
$P_{1} / \mathrm{CCT}$ & $0.43 \pm 0.05$ & $0.44 \pm 0.06$ & $0.47 \pm 0.07$ \\
$\mathrm{HM}$ & $1.31 \pm 0.04$ & $1.33 \pm 0.05$ & $1.31 \pm 0.06$ \\
\hline
\end{tabular}

$\mathrm{T}$ a b $1 \mathrm{e}$ 4. The results of the Mann-Whitney test ( $p$-values) comparison of medians between two groups for the output parameters of the ORA (IOPg, CH, CRF) and those calculated from the raw ORA data (HM, $\left.P_{1} / \mathrm{CCT}, P_{\mathrm{a}} / \mathrm{CCT}, P_{\mathrm{a}} / \mathrm{smm}, \mathrm{dm}, b_{1.45}\right)$. Statistically significant results $(p<0.05)$ are shown in bold font.

\begin{tabular}{llll}
\hline & Normal $v s$. glaucoma & Normal $v s$. suspect & Suspect $v s$. glaucoma \\
\hline IOPg & 0.580 & $\mathbf{0 . 0 1 0}$ & $\mathbf{0 . 0 0 4}$ \\
$\mathrm{CH}$ & 0.960 & $\mathbf{0 . 0 0 3}$ & $<\mathbf{0 . 0 0 1}$ \\
$\mathrm{CRF}$ & 0.952 & $<\mathbf{0 . 0 0 1}$ & $<\mathbf{0 . 0 0 1}$ \\
$P_{\mathrm{a}} / \mathrm{smm}$ & $\mathbf{0 . 0 0 2}$ & $<\mathbf{0 . 0 0 1}$ & $\mathbf{0 . 0 0 2}$ \\
$b_{1.45}$ & $\mathbf{0 . 0 1 0}$ & $<\mathbf{0 . 0 0 1}$ & $\mathbf{0 . 0 0 2}$ \\
$P_{\mathrm{a}} / \mathrm{CCT}$ & 0.060 & $\mathbf{0 . 0 0 3}$ & $\mathbf{0 . 0 3 0}$ \\
$P_{1} / \mathrm{CCT}$ & $\mathbf{0 . 0 2 0}$ & $<\mathbf{0 . 0 0 1}$ & $\mathbf{0 . 0 0 7}$ \\
$\mathrm{HM}$ & 0.750 & $\mathbf{0 . 0 0 5}$ & $\mathbf{0 . 0 0 4}$ \\
\hline
\end{tabular}

The mean values and standard deviations for the parameters calculated from ORA raw data are shown in Table 3.

The results of comparing two independent samples using the Mann-Whitney test for the proposed parameters $\left(\mathrm{HM}, P_{1} / \mathrm{CCT}, P_{\mathrm{a}} / \mathrm{CCT}, P_{\mathrm{a}} / \mathrm{smm}, \mathrm{dm}, b_{1.45}\right)$ and the original parameters from the ORA device (IOPg, $\mathrm{CH}, \mathrm{CRF}$ ) are shown in Table 4.

For IOPg, CH and CRF significant differences $(p<0.05)$ were found between three pairs of considered groups. Significant differences were found between suspect and normal, suspect and glaucoma groups. Higher discrimination between the groups was achieved with $P_{\mathrm{a}} / \mathrm{smm}, b_{1.45}$ and $P_{1} / \mathrm{CCT}$ where significant differences were recorded for three considered groups. No significant differences were found between the normal and glaucoma group for IOPg, CH, CRF, $P_{\mathrm{a}} / \mathrm{CCT}$ and HM.

Next, the Pearson correlation coefficient $r$ between the proposed parameters HM, $P_{1} / \mathrm{CCT}, P_{\mathrm{a}} / \mathrm{CCT}, P_{\mathrm{a}} / \mathrm{smm}, \mathrm{dm}, b_{1.45}$ and IOPg and $\mathrm{CH}$ was assessed. Parameters $P_{\mathrm{a}} / \mathrm{CCT}$ $(r=0.91, p<0.05)$ and $P_{1} / \mathrm{CCT}(r=0.90, p<0.05)$ are strongly correlated with the intraocular pressure IOPg. The interesting results were obtained for the parameters $P_{\mathrm{a}} / \mathrm{smm}$ and $b_{1.45}$, because they do not show such strong linear relationships with IOPg. They are less related to the intraocular pressure, so they could represent some other eye properties, indirectly related to IOPg. The Pearson correlation coefficient between $P_{\mathrm{a}} / \mathrm{smm}$ and IOPg is $r=0.69(p<0.05)$, and between $b_{1.45}$ and IOPg it is $r=0.75$ 
$(p<0.05)$. Analysis showed that the parameters $\left(P_{1} / \mathrm{CCT}, P_{\mathrm{a}} / \mathrm{CCT}, P_{\mathrm{a}} / \mathrm{smm}, \mathrm{dm}, b_{1.45}\right)$ are not correlated with $\mathrm{CH}(p>0.05)$.

\section{Discussion}

Some ocular diseases have a mechanical etiology and these include some types of glaucoma, keratoconus and Fuchs corneal dystrophy. The explanation of causes of those pathologies requires the knowledge of mechanical properties of eye tissues. This knowledge include both the physiological phenomenon taking place within the eyeball and the effects of the diagnostic actions, one of which is the procedure of the intraocular pressure measurement.

The Ocular Response Analyzer is a commercially available clinical instrument that has been proposed to characterize corneal biomechanical responses using the noncontact tonometry process. The $\mathrm{CH}$ is considered an indicator of corneal viscosity and $\mathrm{CRF}$ is considered an indicator of the overall resistance of the cornea (corneal rigidity). Recently, 37 parameters were derived from the ORA software allowing a detailed analysis of the corneal deformation signal. Several research studies have investigated the clinical relevance of some of these parameters and reported they could be more useful in diagnosis in some kind of the corneal ectasia and prognosis after refractive surgery treatments $[\underline{11}, \underline{18}, \underline{19}, \underline{23}, \underline{24}]$.

Additionally, the device provides an access to raw data of both air pressure and applanation curves allowing application of different smoothing procedures to the raw data during numerical processing in order to evaluate air pressure and applanation curves in healthy eyes, glaucoma suspects and glaucoma [25]. Based on this analysis, we have proposed new parameters $\left(\mathrm{HM}, P_{1} / \mathrm{smm}, P_{\mathrm{a}} / \mathrm{smm}, \mathrm{dm}, b_{1.45}\right)$ that describe the interrelations between the two curves. The importance of these new parameters can be verified by numerical analysis of their repeatability in different measurements carried out on one eye. Our examinations showed that even though some of these parameters vary for different measurements, they are characterized by a specific type of variability for an individual eye. The obtained results show that some of the parameters indicate a very high repeatability for four measurements on one eye, with the ICC coefficient higher than 0.95 and CV of about $10 \%$. Such ICC values are significantly higher and the values of $\mathrm{CV}$ are lower than those given by typical parameters from the ORA such as IOPg and $\mathrm{CH}$.

Finally, this work sought to determine whether differences in unique Ocular Response Analyzer parameters ( $\mathrm{CH}$ and $\mathrm{CRF}$ ) as well as new parameters calculated from raw ORA data (HM, $\left.P_{1} / \mathrm{smm}, P_{\mathrm{a}} / \mathrm{smm}, b_{1.45}\right)$ exist in glaucomatous eyes and in glaucoma suspect eyes independently from IOP value.

Since the introduction of ORA in clinical practice and glaucoma diagnostics, many research studies have been conducted looking for associations between both $\mathrm{CH}$ and $\mathrm{CRF}$ and different parameters like CCT considered as a risk factor for glaucoma, IOP, 
glaucomatous changes in the optic disc or glaucomatous visual field defects $[13, \underline{14}$, 26-28]. AOKI et al. [29] reported a significant correlation between the parameters of the corneal deformation signal (ORA software) and glaucomatous visual field progression. However, according to our knowledge none of those studies propose new parameters for supporting the early glaucoma detection.

Statistical comparative analysis shows that the parameters proposed in this study better differentiate three considered groups (normal, glaucoma suspects and glaucoma group) in comparison to the values of IOPg and $\mathrm{CH}$. The statistically significant importance of $\mathrm{CH}$ and $\mathrm{CRF}$ parameters in glaucoma diagnostics were reported in $[30,31]$. A number of reports have suggested with evidence that $\mathrm{CH}$ is lower in glaucomatous eyes compared with normal eyes and eyes with ocular hyper tension $[\underline{9}, \underline{14}, \underline{15}, \underline{31}, \underline{32}]$. It is known that $\mathrm{CH}$ is influenced by IOP value which means the differentiation between healthy subjects and glaucoma patients could be unclear if patients take medication to regulate IOP level. In this study glaucoma patients were taking medication, therefore the results may be less agreable to some reported in literature $[\underline{14}, \underline{30}, \underline{31}]$. However, Sun et al. [33] reported that even after pharmacologic reduction of IOP, $\mathrm{CH}$ was lower in glaucomatous eye than normal eyes. CRF parameter is more correlated with corneal ectasia and corneal structure degeneration connected with long term use of eye drops or systemic medication [6]. However, most papers presented the comparison of ORA parameters between healthy and glaucomatous eyes; little work has been conducted on glaucoma suspects.

The glaucoma suspects are difficult to diagnose as results are based on intraocular pressure values measured by tonometry. The unique output parameters of ORA and those proposed in this study (calculated from raw data) could make the diagnosis of glaucoma suspects easier and clearer. The results in Table 4 show significant differences for HM, $P_{1} / \mathrm{smm}, P_{\mathrm{a}} / \mathrm{smm}, b_{1.45}$ parameters, the suspect group is statistically distinguishable $(p<0.05)$ from healthy eyes as well as from glaucomatous eyes.

The findings of this study indicate that the analysis of ORA parameters could be utilized in early glaucoma diagnostics and that the parameters calculated from raw ORA data seem to be promising as being more sensitive than others to detect glaucoma. It is worth emphasizing that repeatability of proposed parameters could be even higher (ICC for $P_{\mathrm{a}} / \mathrm{smm}$ is 0.83 and for $\mathrm{dm}$ is 0.86 ) than repeatability of the original ORA parameters (ICC for $\mathrm{CH}$ is 0.69 ) (see Table 2).

The proposed HM parameter describes corneal viscoelasticity and is highly correlated with the $\mathrm{CH}$, however it takes into account the value of the maximal air pressure MP during measurement. While $\mathrm{CH}$ is calculated from $P_{1}$ and $P_{2}$ : air pressures corresponding with two applanation states of the cornea. HM parameter does not differentiate normal and glaucoma groups, similarly as $\mathrm{CH}$. However, repeatability of HM increases for the ratio of $\mathrm{HM}$ and $\mathrm{CCT}$ and then is higher than repeatability of $\mathrm{CH}$. It would be interesting to examine and compare the repeatability of $\mathrm{HM}$ parameter with $\mathrm{CH}$ and their quotients with the corneal thickness on groups of patients with different eye pathologies. Probably this type of parameters carried information about the structure and material parameters of eye tissues that could be used in ophthalmology. 
The relationship between ocular biomechanics and glaucoma is still unknown, but it offers a great potential for further investigations, the results of which may have a substantial impact on future glaucoma diagnosis and treatment.

Acknowledgements - This work was partially supported by the National Science Centre under grant 2016/21/N/ST7/02682.

\section{References}

[1] Koprowski R., Tian L., Quantitative assessment of the impact of blood pulsation on intraocular pressure measurement results in healthy subjects, Journal of Ophthalmology, Vol. 2017, article 9678041, DOI: $10.1155 / 2017 / 9678041$.

[2] Pourjavan S., Boelle P.Y., Detry-Morel M., De Potter P., Physiological diurnal variability and characteristics of the ocular pulse amplitude (OPA) with the dynamic contour tonometer (DCT-Pascal $\left.{ }^{\mathbb{R}}\right)$, International Ophthalmology 27(6), 2007, pp. 357-360, DOI: 10.1007/s10792-007-9161-7.

[3] Hoffmann E.M., Grus F.H., Pfeiffer N., Intraocular pressure and ocular pulse amplitude using dynamic contour tonometry and contact lens tonometry, BMC Ophthalmology 4, 2004, article 4, DOI: 10.1186/1471-2415-4-4.

[4] Doughty M.J., Zaman M.L., Human corneal thickness and its impact on intraocular pressure measures: a review and meta-analysis approach, Survey of Ophthalmology 44(5), 2000, pp. 367-408, DOI: 10.1016/S0039-6257(00)00110-7.

[5] Patwardhan A.A., Khan M., Mollan S.P., Haigh P., The importance of central corneal thickness measurements and decision making in general ophthalmology clinics: a masked observational study, BMC Ophthalmology 8, 2008, article 1, DOI: 10.1186/1471-2415-8-1.

[6] Котесha A., Elsheikn A., Roberts C.R., Zhu H., Garway-Heath D.F., Corneal thickness- and age -related biomechanical properties of the cornea measured with the Ocular Response Analyzer, Investigative Ophthalmology and Visual Science 47(12), 2006, pp. 5337-5347, DOI: 10.1167/iovs. 06-0557.

[7] Asejczyk-Widlicka M., Srodka W., Pierscionek B., A comparative analysis of Goldmann tonometry correction, Journal of Glaucoma 26(3), 2017, pp. 233-240, DOI: 10.1097/IJG.0000000000000588.

[8] Hamilton K.E., Pye D.C., Young's modulus in normal corneas and the effect on applanation tonometry, Optometry and Vision Science 85(6), 2008, pp. 445-450, DOI: 10.1097/OPX.0b013e3181783a70.

[9] LuCE D.A., Determining in vivo biomechanical properties of the cornea with an ocular response analyser, Journal of Cataract and Refractive Surgery 31(1), 2005, pp. 156-162, DOI: $10.1016 / \mathrm{j} . j \mathrm{crs}$. 2004.10.044.

[10] Bonatti J.A., Bechara S.J., Carricondo P.C., Kara-José N., Proposal for a new approach to corneal biomechanics: dynamic corneal topography, Arquivos Brasileiros de Oftalmologia 72(2), 2009, pp. 264-267, DOI: 10.1590/s0004-27492009000200028.

[11] Touboul D., Bénard A., Mahmoud A.M., Gallois A., Colin J., Roberts C.J., Early biomechanical keratoconus pattern measured with an ocular response analyzer: curve analysis, Journal of Cataract and Refractive Surgery 37(12), 2011, pp. 2144-2150, DOI: 10.1016/j.jcrs.2011.06.029.

[12] Pepose J.S., Feigenbaum S.K., Qazi M.A., Sanderson J.P., Roberts C.J., Changes in corneal biomechanics and intraocular pressure following LASIK using static, dynamic, and noncontact tonometry, American Journal of Ophthalmology 143(1), 2007, pp. 39-47, DOI: 10.1016/j.ajo.2006.09.036.

[13] Murphy M.L., Pokrovskaya O., Galligan M., O’Brien C., Corneal hysteresis in patients with glaucoma-like optic discs, ocular hypertension and glaucoma, BMC Ophthalmology 17, 2017, article 1, DOI: $10.1186 / \mathrm{s} 12886-016-0396-9$.

[14] Congdon N.G., Broman A.T., Bandeen-Roche K., Grover D., Quigley H.A., Central corneal thickness and corneal hysteresis associated with glaucoma damage, American Journal of Ophthalmology 141(5), 2006, pp. 868-875, DOI: 10.1016/j.ajo.2005.12.007. 
[15] Sullivan-Mee M., Billingsley S.C., Patel A.D., Halverson K.D., Alldredge B.R., Qualls C., Ocular response analyzer in subjects with and without glaucoma, Optometry and Vision Science 85(6), 2008, pp. 463-470, DOI: 10.1097/OPX.0b013e3181784673.

[16] KidA T., Liu J.H.K., WeINREB R.N., Effect of 24-hour corneal biomechanical changes on intraocular pressure measurement, Investigative Ophthalmology and Visual Science 47(10), 2006, pp. 4422-4426, DOI: $10.1167 /$ iovs.06-0507.

[17] Avetisov S.E., Novikov I.A., Bubnova I.A., Antonov A.A., Siplivyi V.I., Determination of corneal elasticity coefficient using the ORA database, Journal of Refractive Surgery 26(7), 2010, pp. 520-524, DOI: 10.3928/1081597X-20091030-01.

[18] Kerautret J., Colin J., Touboul D., Roberts C., Biomechanical characteristics of the ectatic cornea, Journal of Cataract and Refractive Surgery 34(3), 2008, pp. 510-513, DOI: 10.1016/j.jcrs.2007.11.018.

[19] Zarei-Ghanavati S., Ramirez-Miranda A., Yu F., Hamilton D.R., Corneal deformation signal waveform analysis in keratoconic versus post-femtosecond laser in situ keratomileusis eyes after statistical correction for potentially confounding factors, Journal of Cataract and Refractive Surgery 38(4), 2012, pp. 607-614, DOI: 10.1016/j.jcrs.2011.11.033.

[20] Elsheikh A., Joda A., Abass A., Garway-Heath D., Assessment of the ocular response analyzer as an instrument for measurement of intraocular pressure and corneal biomechanics, Current Eye Research 40(11), 2015, pp. 1111-1119, DOI: 10.3109/02713683.2014.978479.

[21] Кotecha N., White E., Schlottmann P.G., Garway-Heath D.F., Intraocular pressure measurement precision with the Goldmann applanation, dynamic contour, and ocular response analyzer tonometers, Ophthalmology 117(4), 2010, pp. 730-737, DOI: 10.1016/j.ophtha.2009.09.020.

[22] JóźwiK A., KASPRZAK H., KuCZMA M., New approach to the analysis of raw data from the Ocular Response Analyzer, Biomedicine Hub 1(3), 2016, article 452124, DOI: 10.1159/000452124.

[23] Spoerl E., Terai N., Scholz F., Raiskup F., Pillunat L.E., Detection of biomechanical changes after corneal cross-linking using Ocular Response Analyzer software, Journal of Refractive Surgery 27(6), 2011, pp. 452-457, DOI: 10.3928/1081597X-20110106-01.

[24] Sedaghat M., Naderi M., Zarei-Ghanavati M., Biomechanical parameters of the cornea after collagen crosslinking measured by waveform analysis, Journal of Cataract and Refractive Surgery 36(10), 2010, pp. 1728-1731, DOI: 10.1016/j.jcrs.2010.06.056.

[25] Asejczyk-Widlicka M., Jóźwik A., Kasprzak H., Sobczak M., Pierścionek B., Data analysis of the Ocular Response Analyzer for improved distinction and detection of glaucoma, Journal of the Optical Society of America A 36(4), 2019, pp. B71-B76, DOI: 10.1364/JOSAA.36.000B71.

[26] Anand A., De Moraes C.G.V., Teng C.C., Tello C., Liebmann J.M., Ritch R., Corneal hysteresis and visual field asymmetry in open angle glaucoma, Investigative Ophthalmology and Visual Science 51(12), 2010, pp. 6514-6518, DOI: 10.1167/iovs.10-5580.

[27] Prata T.S., Lima V.C., Guedes L.M., Biteli L.G., Teixeira S.H., de Moraes C.G., Ritch R., Paranhos Jr A., Association between corneal biomechanical properties and optic nerve head morphology in newly diagnosed glaucoma patients, Clinical and Experimental Ophthalmology 40(7), 2012, pp. 682-688, DOI: 10.1111/j.1442-9071.2012.02790.x.

[28] Park K., Shin J., Lee J., Relationship between corneal biomechanical properties and structural biomarkers in patients with normal-tension glaucoma: a retrospective study, BMC Ophthalmology $\mathbf{1 8}$, 2018, article 7, DOI: 10.1186/s12886-018-0673-X.

[29] Aoki S., Murata H., Matsuura M., Fujino Y., Nakakura S., Nakao Y., Kiuchi Y., Asaoka R., The relationship between the waveform parameters from the Ocular Response Analyzer and the progression of glaucoma, Ophthalmology Glaucoma 1(2), 2018, pp. 123-131, DOI: 10.1016/j.ogla. 2018.08.006.

[30] Shah S., Laiquzzaman M., Mantry S., Cunliffe I., Ocular response analyser to assess hysteresis and corneal resistance factor in low tension, open angle glaucoma and ocular hypertension, Clinical and Experimental Ophthalmology 36(6), 2008, pp. 508-513, DOI: 10.1111/j.1442-9071.2008.01828.x. 
[31] Mansouri K., Leite M.T., Weinreb R.N., Tafreshi A., Zangwill L.M., Medeiros F.A., Association between corneal biomechanical properties and glaucoma severity, American Journal of Ophthalmology 153(3), 2012, pp. 419-427, DOI: 10.1016/j.ajo.2011.08.022.

[32] Grise-Dulac A., SaAd A., Abitbol O., Febbraro J.L., Azan E., Moulin-Tyrode C., Gatinel D., Assessment of corneal biomechanical properties in normal tension glaucoma and comparison with open-angle glaucoma, ocular hypertension, and normal eyes, Journal of Glaucoma 21(7), 2012, pp. 486-489, DOI: 10.1097/IJG.0b013e318220daf0.

[33] Sun L., Shen M., Wang J., FAng A., Xu A., FAng H., Lu F., Recovery of corneal hysteresis after reduction of intraocular pressure in chronic primary angle-closure glaucoma, American Journal of Ophthalmology 147(6), 2009, pp. 1061-1066, DOI: 10.1016/j.ajo.2009.01.008.

Received February 14, 2019 in revised form March 21, 2019 